COMENTARIO

\title{
Historia e impacto de la literatura científica del Departamento de Madre de Dios, Perú*
}

\author{
History and impact of the scientific literature of the Department of Madre \\ de Dios, Peru
}

\author{
Nigel C. A. Pitman ${ }^{1,2}$, Karina Salas ${ }^{1,3}$, María del Carmen Loyola Azáldegui ${ }^{4}$, \\ Gabriela Vigo ${ }^{5}$ y David A. Lutz ${ }^{6}$
}

1. Autor correspondiente: Asociación para la Conservación de la Cuenca Amazónica, Jirón 499, Puerto Maldonado, Madre de Dios, Peru, email Nigel Pitman: npitman@amazonconservation.org 2. Center for Tropical Conservation, Nicholas School of the Environment and the Earth Sciences, Box 90381 Duke University, Durham, NC 27708-0381 USA

3. Universidad Nacional San Antonio Abad del Cusco (sede Puerto Maldonado), Jirón San Martín Cuadra 4, Puerto Maldonado, Madre de Dios, Perú

4. Centro de Datos para la Conservación - CDC, Universidad Nacional Agraria La Molina UNALM. Av. La Molina s/n , La Molina Lima Perú.

5. Facultad de Ciencias, Universidad Nacional Agraria La Molina, Avenida La Molina s/n, La Molina, Lima, Perú

Asociación para la Conservación de la Cuenca Amazónica, Avda. Oswaldo Baca 402, Urbanización Magisterio, Cusco, Perú.

Nota del Editor: el presente trabajo es la version actualizada y en español de Pitman et al. 2007 Publicado por la relevancia del tema en la politica editorial de la Rev peru biol.

Presentado: 09/06/2008 Aceptado: 19/08/2008 Publicado online: 26/02/2009

\section{Resumen}

Los libros, artículos, documentos gubernamentales y otros registros escritos de la biología y conservación de los trópicos llegan a solo una pequeña fracción de sus lectores potenciales. Algunos de los textos son inaccesibles debido al idioma en que están escritos. Otros sólo están disponibles a subscriptores de revistas caras del mundo desarrollado, o circulan exclusivamente en los países tropicales. Para entender mejor esta ineficiencia en la literatura científica tropical y lo que pueda significar para la conservación, realizamos una recopilación bibliográfica de las obras relacionadas a biología y conservación del departamento amazónico de Madre de Dios, Perú. El esfuerzo dio como resultado una lista de 2.202 textos escritos entre 1537 y 2004 , sumando aproximadamente 80.000 páginas. El 93\% de estos textos fueron escritos a partir de 1970. En 2004 la tasa de publicación había alcanzado casi tres textos por semana. Aproximadamente la mitad de la bibliografía de Madre de Dios es representada por textos en español escritos por autores peruanos y difíciles de conseguir fuera del Perú; la mayoría del resto son textos escritos en inglés por autores extranjeros y difíciles de conseguir en el Perú. Los autores extranjeros tienden a escribir acerca de estudios ecológicos con poca relevancia a los retos inmediatos de la conservación, mientras los autores peruanos hacen con mayor frecuencia recomendaciones prácticas para el manejo. En la lista de temas más tratados por estos textos, el uso sostenible de recursos naturales figura en tercer lugar, después de la ecología y el comportamiento animal. Se concluye el documento con algunas recomendaciones para convertir a la literatura tropical en un recurso más abierto y eficiente.

Palabras claves: Conservación, cuenca amazónica, literatura científica, Madre de Dios, Perú, publicaciones

\section{Abstract}

Books, articles, government documents, and other written accounts of tropical biology and conservation reach a tiny fraction of their potential audience. Some texts are inaccessible because of the language in which they are written. Others are only available to subscribers of developed-world journals, or distributed narrowly within tropical countries. To examine this dysfunction in the tropical literature-and what it means for conservationwe spent a year trying to compile everything ever written on the biology and conservation of the department of Madre de Dios, Peru, in southwestern Amazonia. Our search of libraries, databases, and existing bibliographies uncovered 2202 texts totaling roughly 80000 pages. Texts date from 1553 to 2004 , but $93 \%$ were written after 1970. Since that year the publication rate has increased steadily from fewer than ten texts per year to nearly three texts per week in 2004. Roughly half of the Madre de Dios bibliography is accounted for by Spanishlanguage texts written by Peruvian authors and mostly inaccessible outside Peru; most of the remainder are English-language texts written by foreign authors and largely inaccessible in Peru. Foreign authors tended to write about ecological studies with limited relevance to on-the-ground conservation challenges, while Peruvian authors were more likely to make specific management recommendations. In the list of most written-about topics, sustainable use of natural resources ranks third behind ecology and animal behavior. We conclude with some recommendations for converting the tropical literature to a more open and efficient resource for science and conservation.

Keywords: Conservation, Amazon basin, literature, Madre de Dios, Peru, publications

\section{Introducción}

Aquello que los biólogos escriben hoy acerca de los bosques tropicales será, en muchos casos, lo único que quede de ellos en el futuro. El bosque ecuatoriano donde hace 20 ańos se registró el record mundial en diversidad de anuros fue destruido poco después, pero un joven herpetólogo aún puede visitarlo hoy a través de los artículos científicos (Duellman 1978, 1988; Pitman et al. 2002). Los documentos escritos también son los principales hitos para medir el avance del conocimiento científico sobre la naturaleza tropical, así como un medio importante para comunicar esos avances a las personas que manejan, estudian, o habitan los ecosistemas tropicales. Por consiguiente, el éxito de la campańa de prevenir extinciones masivas en los trópicos durante el siglo XXI dependerá en gran medida de la calidad de la comunicación escrita.

Pero la literatura científica tropical sufre de graves problemas. Mucho de aquello que se escribe sobre los trópicos se publica en inglés en revistas de países desarrollados, dónde es inaccesible a la mayoría de los biólogos tropicales (Primack 2001). Por ejemplo, la primera versión del presente artículo fue publicada en inglés en Conservation Biology (Pitman et al. 2007). Para poder leerlo por las vías oficiales, un estudiante universitario peruano tendría que pagar US\$39 (el precio de comprar el artículo en formato pdf), pagar US\$50 (el costo de suscribirse por un año), o convencer a su universidad a pagar US\$730 (el precio de una suscripción institucional). Existen obstáculos similares para los biólogos tropicales en países desarrollados, ya que muchos conocimientos científicos valiosos son difundidos exclusivamente dentro de los países tropicales en portugués, español, francés y otros idiomas.

El material considerado por muchos como la crema de la literatura tropical -los artículos publicados en inglés por revistas peer review en países desarrollados- padece de otros prejuicios bien documentados. Por ejemplo, muy pocos autores de estos artículos son ciudadanos de países tropicales (Fazey et al. 2005a, b; 
Holmgren \& Schnitzer 2004). Muy pocos artículos tratan la biología de invertebrados, prefiriendo los grandes y carismáticos vertebrados (Clark \& May 2002a, b; Fazey et al. 2005a). Los artículos peer review son publicados en promedio cuatro años después de que los datos fueran colectados (Fazey et al. 2005a). Contienen recomendaciones que los autores consideran útiles para el manejo de las áreas protegidas (Flaspohler et al. 2000; Ormerod et al. 2002) pero que los responsables de las áreas protegidas generalmente ignoran (Pullin et al. 2004).

Dado que el contenido y la calidad de la literatura tropical tienen consecuencias de gran envergadura para la conservación global, estas ineficiencias merecen más atención. Para entenderlas mejor, en este artículo examinamos todo lo escrito sobre la biología y conservación del departamento peruano de Madre de Dios. Basamos nuestro estudio en las siguientes preguntas sencillas: ¿Quiénes escribieron estos textos? ¿Sobre cuáles temas, organismos, y acerca de cuáles lugares escribieron? ¿En cuál formato, en cuál idioma, y con cuánto éxito se difundieron los textos escritos? ¿Hasta qué punto los textos fueron útiles para el manejo de las áreas naturales de Madre de Dios? ¿Qué clase de registro histórico están dejando los biólogos tropicales sobre estos bosques? Y finalmente, ¿¿dónde se puede identificar ineficiencias y qué se puede hacer para corregirlas?

\section{Área de estudio}

El departamento de Madre de Dios ocupa $85183 \mathrm{~km}^{2}$ al pie de los Andes en el sudoeste amazónico. El área ocupada por Madre de Dios es un poco mayor al de Panamá y corresponde aproximadamente al 1\% de la cuenca amazónica. Una quinta parte del departamento es bosque montano situado sobre los $500 \mathrm{~m}$ de altitud. El resto es selva baja, humedales, ríos, lagos, y parches de vegetación dominada por el bambú (Goulding et al. 2003).

\section{Material y métodos}

Para elaborar la bibliografía de Madre de Dios realizamos una búsqueda intensiva en bibliotecas peruanas, así como en bibliografías publicadas y bases de datos en INTERNET. Revisamos las bibliotecas de ocho universidades e institutos superiores (la Universidad Nacional Agraria La Molina, la Universidad Nacional Amazónica de Madre de Dios, la Universidad Nacional Mayor de San Marcos, la Universidad Nacional San Antonio Abad del Cusco sede Puerto Maldonado, la Universidad Peruana Cayetano Heredia, la Universidad Ricardo Palma, el Instituto Superior Tecnológico Jorge Basadre Grohman, y el Instituto Superior Pedagógico Puerto Maldonado), ocho agencias gubernamentales (principalmente el Instituto Nacional de Recursos Naturales -INRENA y el Instituto de Investigaciones de la Amazonía Peruana -IIAP), siete organizaciones no gubernamentales (Asociación para la Conservación de la Cuenca Amazónica, Asociación para la Nińez y su Ambiente, CESVI, Conservación Internacional, ProNaturaleza, Sociedad Zoológica de Frankfurt, TReeS Perú) y algunas empresas de ecoturismo, así como la Biblioteca Nacional del Perú. La mayoría de estas bibliotecas se encuentran en las ciudades de Lima, Cusco, Iquitos, y Puerto Maldonado. Incorporamos las bibliografías existentes de Wahl Kleiser y Rummenhoeller (1991), Leite Pitman et al. (2003), y de las estaciones biológicas de Cocha Cashu y Los Amigos. Las bases de datos de INTERNET más utilizadas durante la elaboración de la bibliografía fueron ISI Web of Science y Google Scholar.
Durante estas búsquedas recopilamos la información bibliográfica de todo artículo de revista, libro, volumen editado, capítulo de volumen editado, tesis, e informe no publicado que contuviera información sobre la biología o conservación de Madre de Dios. Además de los campos bibliográficos típicos (autor o editor, año, título, tipo de referencia, número de páginas, imprenta, ciudad de imprenta), también se registró el idioma de cada referencia. Una vez que todas las referencias fueron incluidas en una base de datos, se asignó a cada referencia palabras claves para indicar el campo de estudio (e.g., ecología, taxonomía, antropología, manejo de áreas naturales protegidas), las áreas de interés (e.g., interacciones planta-animal, etnobotánica, comportamiento reproductivo), los organismos estudiados y el sitio de estudio. Las palabras claves para éste último incluyeron hábitat (e.g., dosel de bosque, hábitats acuáticos, pantanos), áreas protegidas y la escala espacial. Para cada referencia también se registró la nacionalidad del primer autor, cuando ésta era mencionada o conocida. Las palabras claves y nacionalidades fueron asignadas por los autores basados en la información bibliográfica, en los resúmenes y en la experiencia personal.

Restringir la bibliografía a la biología y conservación significó tomar algunas decisiones arbitrarias. Los textos que trataban de geografía, geología, suelos, y clima fueron incluidos, mientras los documentos que trataban de la actividad agrícola, maderera, minería, salud pública, medicina, historia, o poblaciones indígenas sólo fueron considerados cuando contaban con información biológica significativa o trataban sobre alguna área protegida. Una completa bibliografía científica de la región tendría necesariamente que incluir la descripción taxonómica de cada especie registrada en Madre de Dios, así como las revisiones y tratamientos subsecuentes, sin embargo una recopilación de esa escala no era factible. Por lo tanto la bibliografía sólo incluye una porción pequeña de toda la literatura taxonómica relevante a Madre de Dios.

\section{Resultados}

\section{Resultados generales}

La búsqueda resultó en una bibliografía de 2202 textos, los cuales suman aproximadamente 80000 páginas. Los artículos en revistas fueron el tipo de texto más común, pero los informes no publicados representaron el mayor número de páginas (Figuras 1 a y b). Casi todos los textos estaban escritos en español $(54,9 \%)$ o inglés $(43,6 \%)$, siendo el tercer idioma más común el alemán (0,5\%; Figura 1c). El 90\% de los textos escritos por autores peruanos estaban en espańol, y el $85 \%$ de los textos escritos por autores norteamericanos estaban en inglés. Por cada 46 artículos que los autores norteamericanos publicaron sobre ecología en inglés, publicaron un artículo sobre ecología en espańol. En contraste, menos del $20 \%$ de los textos relacionados a ecología escritos por autores peruanos fueron artículos de revistas, y la mayoría de éstos en español. Los autores europeos fueron los más equitativos en lo que se refiere al idioma; el $60 \%$ de sus textos estaban en inglés, el $31 \%$ en español y el resto en otros idiomas.

La bibliografía data de 455 años atrás, pero dos terceras partes de los documentos tienen menos de 15 años de antigüedad y sólo el 7\% data antes de 1970. Los documentos más antiguos describen la primera visita europea a la región en 1553 (Cieza de León 1877) y la segunda en 1567 (Ulloa 1899). Después de 

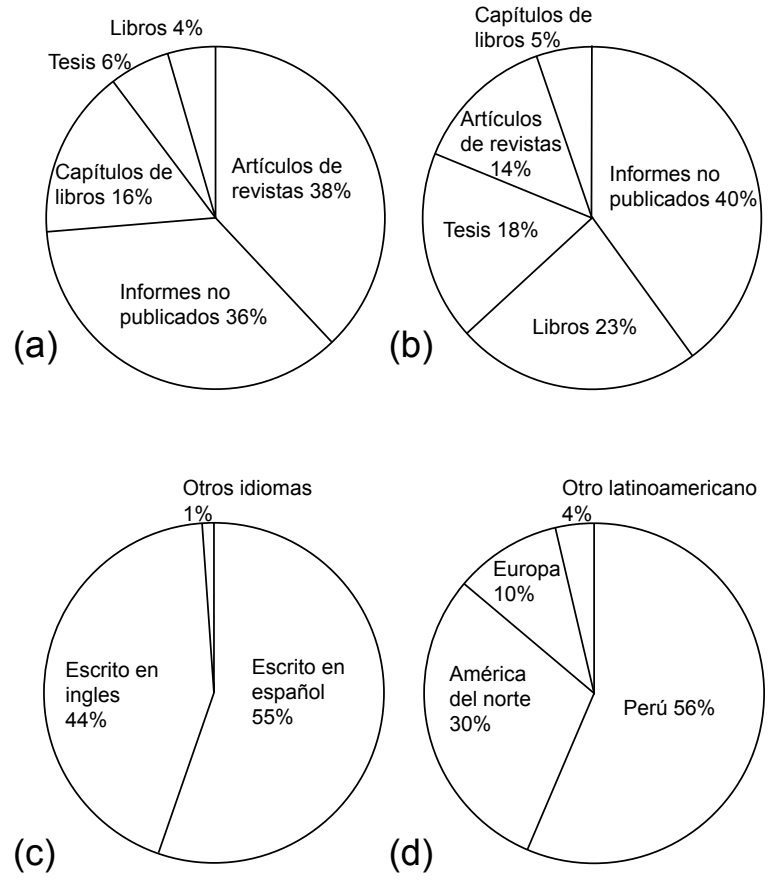

Figura 1. a. Proporción de textos acerca de la biología y conservación de Madre de Dios, por tipo de referencia. b. Proporción de páginas por tipo de referencias. c. Proporción de textos escritos en diferentes idiomas. d. Proporción de textos escritos por autores de diferentes nacionalidades.

estos documentos se abre un silencio de 278 años. El número de las publicaciones aumentó durante el auge de caucho en los últimos años del siglo XIX pero colapsó con el precio del caucho dos décadas después. Al principio de la década de los 70 se inicia un crecimiento explosivo en el número de publicaciones (Fig. 2).

Los 838 artículos científicos en la bibliografía fueron publicados en 276 revistas diferentes. Solamente 20 de éstas han publicado diez o más artículos sobre Madre de Dios. Seis de éstas revistas son peruanas y el resto norteamericanas o europeas. Las diez revistas con el mayor número de artículos son el Boletín de la Sociedad Geográfica de Lima, Misiones Dominicanas del Perú, Biotropica, Revista Peruana de Entomología, Ecology, American Journal of Physical Anthropology, Wilson Bulletin, Condor, Journal of Tropical Ecology, e International Journal of Primatology.

\section{Contenido}

La mayoría de los documentos con fechas anteriores a 1960 son relatos de viaje de exploradores, misioneros y extractores del caucho. El primer documento identificable como un texto biológico moderno es un artículo de 1900 escrito por el mastozoólogo norteamericano J. A. Allen en el Bulletin of the American Museum of Natural History, titulado "On Mammals Collected in Southeastern Peru by Mr. H. H. Keays, with Descriptions of New Species" (Allen 1900). De los textos escritos entre 1900 y 1960 encontramos sólo seis otros de índole estrictamente científico. En los demás textos históricos, la información relevante a los biólogos modernos es principalmente restringida a la etnobotánica, el uso histórico de la tierra y la distribución geográfica de especies económicamente importantes como caucho (Hevea Aubl. spp.) y castaña (Bertholletia excelsa Humb. \& Bonpl.).

Los organismos más estudiados en Madre de Dios según la bibliografía son las plantas $(21,3 \%$ de todos los textos) y los

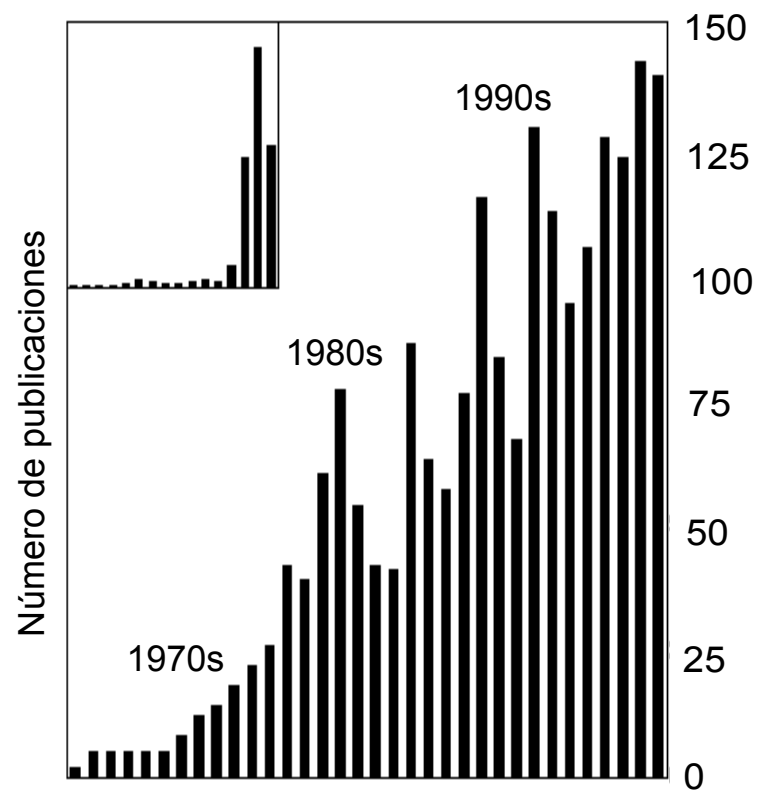

Figura 2. Número de textos sobre la biología y conservación de Madre de Dios, escritos cada año entre 1971 y 2004. La inserción muestra el número de documentos escritos cada década entre 1850 y 2000; la escala del eje vertical en la inserción es de 1000.

mamíferos (16,6\%); de estos predominan los árboles (16,0\%) y primates (7,9\%; Figura 3). Esto se debe en parte a que las tres especies con el mayor número de textos fueron la castańa (6,3\%), el caucho (3,4\%) y los lobos de río (Pteronura brasiliensis Gmelin; 2,9\%). Existen más textos sobre aves (10,6\%) que sobre invertebrados (10,4\%); siguen textos sobre herpetofauna (7,6\%), peces $(2,8 \%)$, y hongos y líquenes (0,3\%). El 97\% de los textos sobre invertebrados versan sobre artrópodos.

El tema más común en la bibliografía es "ecología" (refiriéndose, en este caso, a estudios de organismos en estado silvestre), la cual corresponde a 670 textos y un $30 \%$ del total. Los otros temas comunes son el comportamiento animal ( 435 textos), el uso sustentable de recursos naturales (310) y los productos no maderables (271). La Tabla 1 presenta una lista de las 30 palabras claves temáticas más frecuentes en la bibliografía.

\section{Patrones geográficos}

El $70 \%$ de los textos que tratan la ecología se basa en datos colectados en un solo lugar de Madre de Dios. El 11\% se basa en datos colectados en varios lugares del departamento y un $19 \%$ en datos colectados dentro y fuera de Madre de Dios. La

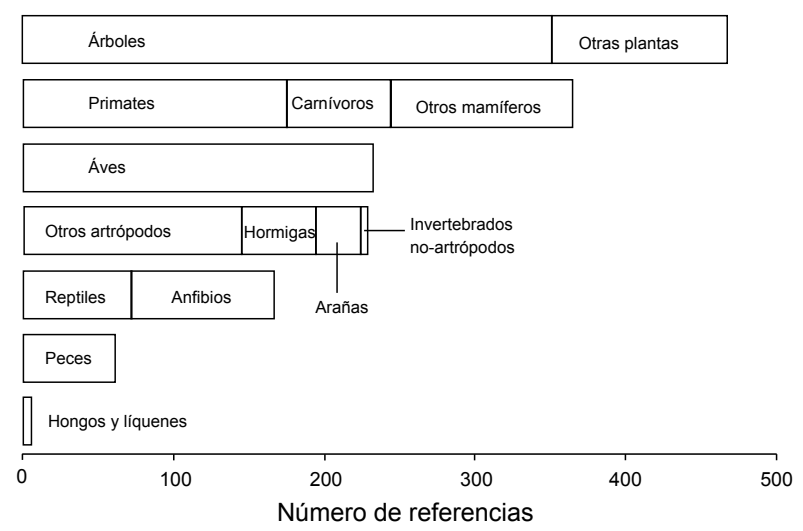

Figura 3. Número de referencias sobre diferentes grupos taxonómicos en la bibliografía de Madre de Dios. 
Tabla 1. Lista de palabras claves temáticas más comunes de la bibliografía de Madre de Dios. Los números entre paréntesis indican el número de referencias que corresponden a cada palabra clave.

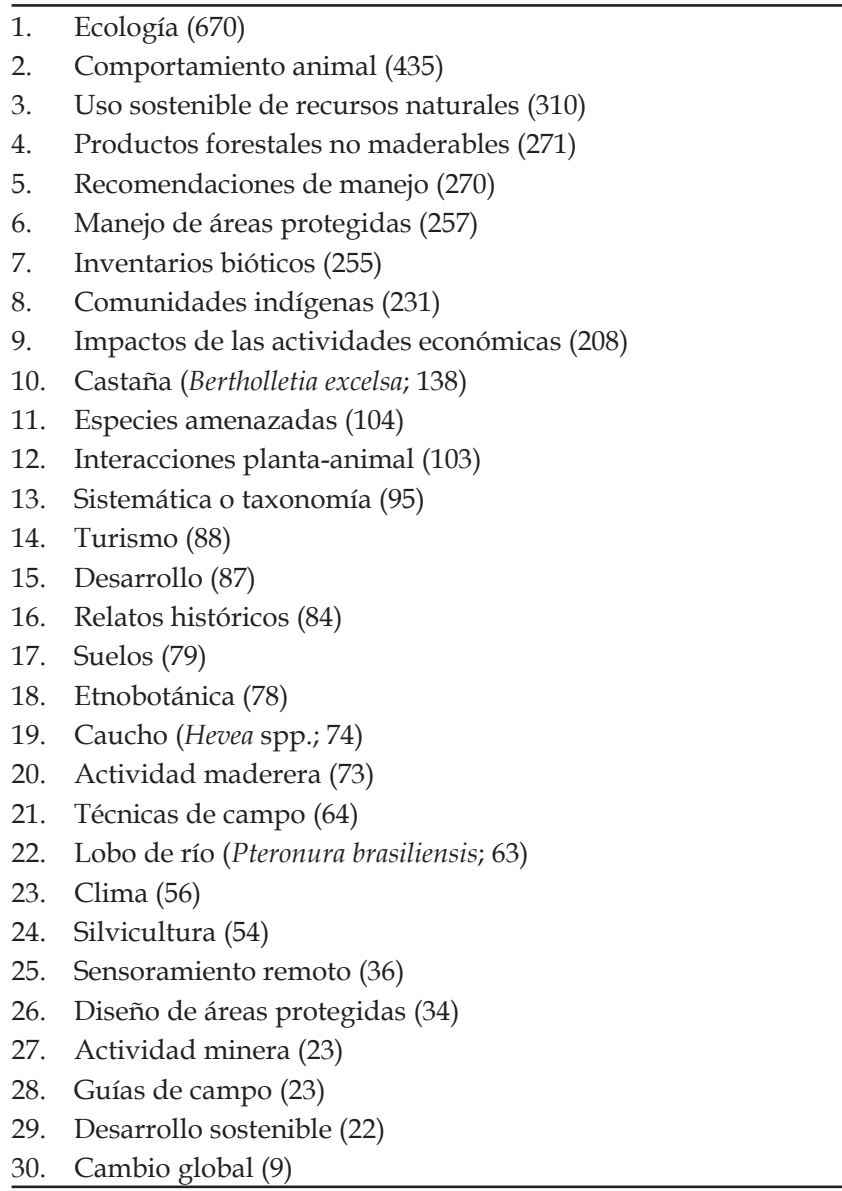

gran mayoría de textos ecológicos describen estudios realizados en bosque primario de suelos bien drenados. Otros hábitats son pobremente representados: hábitats acuáticos (13\% de textos), bosques secundarios (4\%), bosques dominados por bambú (3\%), humedales $(2 \%)$, y dosel del bosque $(<1 \%)$.

El 65\% de los textos en la bibliografía tiene alguna relación a las siete áreas protegidas de la región (Fig. 4). El lugar sobre el cual se ha escrito más en Madre de Dios es la Estación Biológica Cocha Cashu, dentro del Parque Nacional del Manu. Los 420 textos producidos por esta pequeña estación, establecida en 1973, representan casi $20 \%$ de la bibliografía regional, así como el $63 \%$ de los textos sobre la ecología.

\section{Nacionalidades de los autores}

Más de la mitad de los 1791 textos para los cuales la nacionalidad del primer autor es conocida fueron escritos por autores del Perú (56\%) y de otros países sudamericanos (4\%; Fig. 1d). Los autores norteamericanos escribieron el $30 \%$ de los textos y los europeos el 10\%. Diez por ciento de textos fueron distribuidos por instituciones sin indicar el nombre del autor.

Los autores peruanos escribieron el $87,5 \%$ de todos los textos con fechas anteriores al año 1970. Después del incremento de visitas de biólogos extranjeros en los ańos 70, la proporción de textos escritos por los autores peruanos cayó del $84 \%$ en la década de los 70 a tan sólo el $28 \%$ en la siguiente década (Figura 5). Esta proporción llegó a 59\% en la década de los 90 y a $60 \%$ en los años 2000-2004. Los autores peruanos y norteamericanos escribieron sobre temas diferentes y difundieron su trabajo en lu-

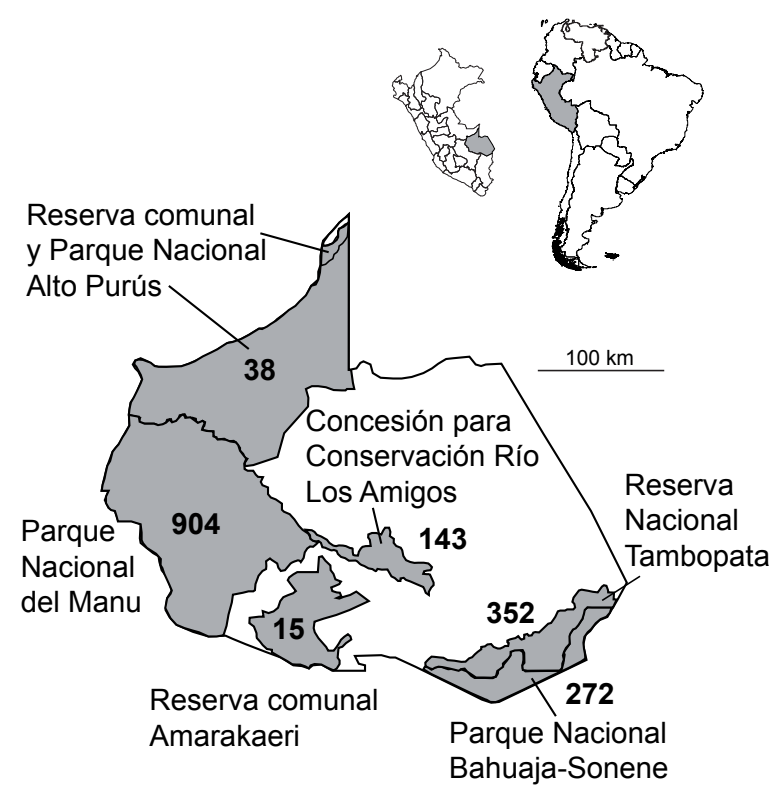

Figura 4. Mapa de áreas protegidas de Madre de Dios, indicando el número de documentos escritos en la bibliografía regional asociados con cada área. La inserción demuestra la localización de Madre de Dios en Sudamérica.

gares diferentes. Los autores norteamericanos escribieron muchos más textos acerca de la ecología y del comportamiento animal y publicaron la mayoría de éstos como artículos en revistas peer review de lengua inglesa. Los autores peruanos ofrecieron muchas más recomendaciones sobre manejo y distribuyeron la mayoría de éstas en informes no publicados escritos en español y distribuidos por el INRENA y las organizaciones no gubernamentales.

\section{Discusión}

Dentro de lo que conocemos, esta es la primera vez que la bibliografía de una región tropical ha sido analizada con el fin de entender mejor quiénes han escrito, acerca de qué temas y dónde acaban esos textos. La mayoría de los resultados no causarán sorpresa en los biólogos experimentados. Algunos reflejan tendencias ampliamente documentadas en la literatura científica de los países desarrollados (Clark \& May 2002b; Fazey et al. 2005a). Otros son bien conocidos por los biólogos que trabajan en los trópicos. La única verdadera sorpresa es que tantos biólogos y conservacionistas hayan soportado una situación tan ineficiente por tanto tiempo. Los que sueńan con el avance de la conservación y la ciencia tropical deben añadir a su larga lista de tareas corregir la manera terriblemente ineficaz con la cual los conservacionistas y biólogos tropicales compartimos la información.

El presente análisis de la bibliografía sobre Madre de Dios desmiente las tres afirmaciones mencionadas con más frecuencia acerca de la literatura tropical: 1) que es escasa, 2) que en su mayoría esta escrita en inglés y por biólogos que no son de países tropicales, y 3) que la información más útil para los responsables de áreas protegidas se publica en las revistas de países desarrollados. Empezamos esta sección tratando estos mitos en más detalle, para después presentar algunas recomendaciones para mejorar la eficacia de la literatura tropical a corto y largo plazo.

\section{Mito 1: La literatura científica tropical es escasa.}

Se invita a los lectores que todavía creen esto a que pasen los próximos ocho meses leyendo los trabajos reunidos sobre Madre de Dios. Ese sería el tiempo necesario, leyendo una página por minuto, 10 horas diarias, para repasar todo el material escrito 


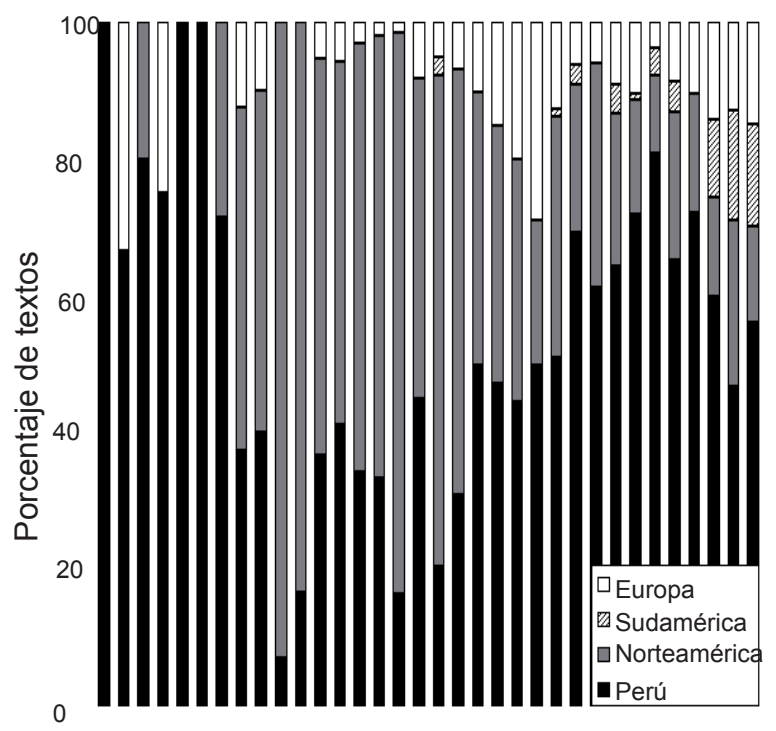

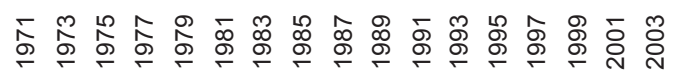

Año

Figura 5. Proporción de textos escritos sobre biología y conservación de Madre de Dios por autores de diferentes nacionalidades entre 1971 y 2004.

hasta la fecha. En el 2004 un nuevo documento sobre Madre de Dios fue lanzado cada dos días y medio, en promedio (Fig. 2). Lejos de ser escasa, la literatura regional es cada vez más voluminosa, habiendo aumentado de forma constante desde 1970. El caso es probablemente igual para la bibliografía de la cuenca amazónica y del Neotrópico en general. Debido a que Madre de Dios es una zona bien estudiada de la Amazonía, no es posible extrapolar un número total de textos escritos acerca de la biodiversidad y conservación en toda la cuenca amazónica. De acuerdo con nuestros resultados en Madre de Dios, consideramos el resultado de 20000 textos una estimación conservadora.

A pesar de esto, la literatura de Madre de Dios todavía parece escasa, ya que aproximadamente la mitad de sus textos son, prácticamente hablando, invisibles. Un estudiante que recorre las bibliotecas de un país desarrollado y un estudiante que recorre las bibliotecas peruanas puede cada uno localizar centenares de documentos acerca de la biodiversidad de la región, pero sus bibliografías tendrán poco en común. Una representará la mitad de la literatura que se publica internacionalmente y en inglés (la llamada "literatura blanca"), mientras la otra representará lo que se publica en el Perú y en espańol (la llamada "literatura gris"). Vale la pena indicar que ni las asombrosas herramientas bibliográficas disponibles ahora en el INTERNET serán capaces de solucionar el problema en el futuro próximo, pues no incluyen a la literatura gris y no proporcionan acceso a la literatura blanca a los usuarios que no cuentan con suscripciones.

También vale la pena mencionar que la literatura tropical sigue siendo escasa desde el punto de vista de uno de sus públicos más importantes: los que no son biólogos. Menos del 1\% de los textos que compilamos se dirige hacia estas personas. Para un colegial de ocho años quien vive en Madre de Dios y se pregunta por primera vez acerca de los pájaros que vuelan sobre su cabeza, o para un periodista en Puerto Maldonado quien escribe un artículo popular acerca de los bosques del departamento, la literatura de la región no ofrece mucha ayuda.
Mito 2: La mayoría de la literatura cientifica tropical está en inglés, escrita por científicos extranjeros y publicada en revistas de países desarrollados.

Ésta es una queja comúnmente escuchada en las universidades tropicales, donde los estudiantes y profesores tienen un acceso limitado a la literatura científica de los países desarrollados. Y es cierto que en las revistas científicas de los países desarrollados la mayoría de los artículos escritos sobre la naturaleza tropical, por lo menos en las revistas de biología de conservación, es escrita por científicos establecidos fuera del Neotrópico (Fazey et al. 2005b). Sin embargo, nuestros datos no apoyan este mito (vea las Figuras 1c y 1d). ¿Por qué persiste?

Una razón es que 18 de los 22 autores más prolíficos (y mejor conocidos) en la bibliografía de Madre de Dios son extranjeros quienes principalmente escriben en inglés: John Terborgh (primer autor de 56 documentos), Charles Janson (37), Scott Robinson (24), Oliver Phillips (22), Robin Foster (18), Patricia Wright (18), Dinah Davidson (17), Alwyn Gentry (17), Christof Schenck (17), Charles Munn (16), Jessica Groenendijk (15), Richard Kiltie (15), William Duellman (14), Terry Erwin (14), Chris Kirkby (14), Ann Rypstra (14), Louise Emmons (13) y Glenn Shephard, Jr. (13). Estos autores también son responsables por una proporción grande de los textos relacionados a ecología, lo cuál significa que los biólogos de nivel universitario en el Perú que no leen inglés sufren una desventaja grave.

Otra razón para la persistencia de este mito es que incluso los documentos escritos sobre Madre de Dios en español y por autores peruanos son difíciles de conseguir en el Perú y aun más en Madre de Dios. Una proporción grande de la literatura de Madre de Dios es accesible solamente en las bibliotecas de unas pocas universidades, museos, gobiernos, y ONGs en Lima y Cusco, las cuales no siempre están abiertas al público en general. Los muchos libros sobre Madre de Dios se venden muy poco en el Perú y no siempre son depositados en las principales bibliotecas peruanas. Al contrario, muchos textos son distribuidos dentro de un círculo pequeño de personas como regalos a colegas, funcionarios públicos, y personal de las ONGs de conservación. Esta práctica garantiza que la mayoría del tiraje de un libro o informe termine en bibliotecas privadas pocos años después de su publicación, en lugar de permanecer accesible para todos en bibliotecas públicas.

Mito 3: Las revistas peer review de los Estados Unidos y Europa publican la crema de la información acerca de la conservación tropical, mientras que la literatura gris contiene información de calidad e importancia menor.

Nuestros datos sugieren que la mayoría de la literatura científica escrita en inglés acerca de Madre de Dios, sea cual fuera su calidad, es esencialmente irrelevante para el manejo de sus siete grandes áreas protegidas y decenas de concesiones de madera, castańa y ecoturismo. Los artículos publicados en las revistas de los países desarrollados muestran muy poca congruencia con los desafíos que enfrentan los responsables de administrar los recursos naturales de Madre de Dios, como por ejemplo: 1) controlar la tala y la caza ilegal (Kometter et al. 2004; Schulte-Herbrüggen 2003), 2) maximizar el valor económico y minimizar el impacto negativo de las actividades legales en los bosques manejados (Flores \& Ashton 2000; Ortiz 2002; Peres et al. 2003; Yu et al. 1997), 3) minimizar los impactos negativos de la pavimentación 
de la Carretera Interoceánica (Dourojeanni 2003; Pautrat 2001), 4) monitorear las comunidades bióticas a través del tiempo para acompañar el estado de conservación de las especies amenazadas o claves de la región (Kirkby 2004), y 5) resolver las aspiraciones a veces contradictorias de los conservacionistas, las comunidades indígenas, y los intereses económicos en el contexto de las áreas protegidas (e.g., Terborgh 1999).

En el período 2000-2004, sólo 19 de los 131 artículos publicados sobre Madre de Dios en los países desarrollados $(<15 \%)$ directamente trataron uno de estos temas. El plan de manejo del área protegida más famosa de la región, el Parque Nacional del Manu, proporciona evidencia adicional de la división profunda entre la literatura científica de alta calidad y las necesidades de los responsables para el manejo de los bosques de la región (INRENA \& PRO-MANU 2004). De las 118 referencias enumeradas en la bibliografía del Plan de Manejo del Manu, sólo 20 provienen de revistas, libros o tesis no peruanos. De éstos 20, sólo siete son relevantes al manejo de las especies o hábitats amenazados en el parque, o a la relación entre el parque y las comunidades indígenas residentes. Se podría argumentar que los autores del plan de manejo tuvieron un limitado acceso a la literatura de los países desarrollados al elaborarlo, pero no fue así; el equipo que supervisó la elaboración del plan incluyó a varios biólogos norteamericanos y europeos.

El hecho de que una fracción tan pequeña, de los centenares de documentos publicados en el extranjero, fue utilizada para el plan de manejo de uno de los parques tropicales más famosos del mundo, debe llamar a reflexión a los biólogos tropicales que asumen en buena fé que sus investigaciones tienen relevancia para la conservación.

Nuestro deseo no es criticar a los investigadores cuyos trabajos no tienen relevancia para la conservación hoy en día, o sugerir que tales trabajos no tienen valor. El punto es pedir a los científicos que investigan los bosques tropicales, sea cual fuera su nacionalidad, que examinen y reexaminen las conexiones entre el trabajo que hacen y el trabajo que hacen los responsables del manejo de las tierras tropicales.

Los lectores que todavía no están convencidos acerca del valor de la literatura gris tropical deben recordar la súplica de Soulé (1985) para que los biólogos de conservación aprovechen toda la información disponible, incorporando los datos incompletos, la incertidumbre, la intuición y la experiencia personal. Mucha de la literatura gris de la Amazonía peruana ha sido escrita por biólogos y conservacionistas cuyo entrenamiento no se ha logrado en las universidades extranjeras sino en el trabajo de campo. Se la puede criticar por la falta de rigor científico, pero contiene datos e ideas sumamente valiosas para el manejo responsable de las tierras tropicales. En el caso de Madre de Dios, un científico tiene que leer la literatura gris si quiere datos sobre, por ejemplo, la cantidad de madera extraída ilegalmente de la región (Pautrat 2003), la cantidad de bosque convertido a otros usos entre 1990 y 2000 (INRENA et al. 2003, 2004), el impacto económico de la industria del ecoturismo en la capital de Madre de Dios (Kirkby 2002), y otra media docena de temas de semejante urgencia.

\section{Recomendaciones}

En un mundo ideal, todo lo escrito acerca de los ecosistemas tropicales sería accesible en archivos digitales y multilingües en el INTERNET. Dado los estupendos avances en la organización de la información durante la última década, tal vez esto no sea una meta tan descabellada para 2020. Mientras tanto, proponemos varios pequeños pasos que podrían tomarse para mejorar el uso y la utilidad de la literatura amazónica durante los próximos años. Usamos la región de la Amazonía como un ejemplo; reformas semejantes deberían implementarse en otras zonas tropicales.

Compilar una bibliografía de la biodiversidad amazónica. Compilar la bibliografía sobre Madre de Dios tomó menos de un año, costó menos de US\$10.000 y fue realizado en gran parte por estudiantes universitarios peruanos. Estimamos un costo similar para compilar una bibliografía que incorpore el resto de la Amazonía peruana. Dado que la gran mayoría de la literatura amazónica reside en un número relativamente pequeńo de ciudades, estimamos el costo de elaborar una bibliografía de toda la cuenca en US\$150.000.

Construir una biblioteca virtual para la Amazonía. La bibliografía de Madre de Dios es ahora accesible en una base de datos en línea que permite a los usuarios realizar búsquedas, descargar resultados de las mismas y agregar nuevas referencias (http://atrium.andesamazon.org/biblio). Dentro de poco será posible descargar de la misma página versiones digitales de los documentos que no sean protegidos por derechos de autor.

Puesto que las exigencias tecnológicas para construir una biblioteca virtual en INTERNET ya no son prohibitivas, la construcción de una para la Amazonía representa más un desafío político e institucional que técnico. La pregunta clave es cómo construir una biblioteca virtual que se mantenga actualizada año tras año y que lo haga de una manera eficiente en términos de costo. Nuestra experiencia en Madre de Dios sugiere que una biblioteca virtual eficaz requerirá de un personal permanente pequeño y un presupuesto anual adecuado. En la Amazonía peruana, queda evidente que la institución mejor posicionada para prestar este servicio es el Instituto de Investigaciones de la Amazonía Peruana (IIAP).

Publicar en INTERNET versiones digitales de nuevos textos, depositando los ejemplares impresos en bibliotecas importantes. Vale la pena recalcar estos pasos obvios porque en nuestra experiencia no son una práctica establecida en las agencias gubernamentales, universidades, y ONGs de conservación en Sudamérica. Las organizaciones de conservación y las instituciones que las financian deberían reconocer que imprimir libros atractivos es una manera muy ineficiente de difundir la información en los países tropicales, a menos que una proporción grande del tiraje sea depositada en las bibliotecas públicas o que versiones digitales sean permanentemente disponibles.

Escanear textos antiguos importantes. Muchos de los documentos más citados en la bibliografía sobre Madre de Dios son libros antiguos que no están disponibles en formato digital (e.g., ONERN 1972, 1977; Terborgh 1983; Gentry 1990). Éstos deben ser escaneados y hechos disponibles en el INTERNET, siempre y cuando los derechos del autor lo permitan.

Invertir en las síntesis, las traducciones, y los resúmenes populares de textos científicos importantes. Para un estudiante de biología en el primer año de universidad en Puerto Maldonado, una bibliografía de 2202 documentos tal vez no sea de mucha ayuda sin un texto que le oriente hacia los docu- 
mentos más valiosos, tal como lo hace una publicación como la Historia Natural de Costa Rica (Janzen 1983). De igual modo, los responsables de áreas de conservación en Madre de Dios no tienen el tiempo para leer la inmensa cantidad de literatura científica que sale publicada sobre la región cada mes, y podrían beneficiarse de textos que sintetizan los avances más relevantes (Fazey et al. 2004; Pullin \& Knight 2001). Finalmente, los textos que proporcionan la mejor descripción actual de ciertos grupos taxonómicos, regiones, o hábitats en la región deberían ser traducidos para que estén disponibles tanto en español como en inglés y portugués (Primack 2001). Esto también es una tarea relativamente barata.

Muy pocos documentos en la bibliografía tratan la historia de la conservación en Madre de Dios. Sin duda, los futuros pobladores del departamento se preguntaran sobre las fuerzas que conllevaron a la declaración de casi la mitad de la región como área protegida en un período de tan solo 35 años. Contar bien esa historia tiene un valor más que académico, pues los residentes de Madre de Dios no seguirán proporcionando apoyo a este tipo de cambios si los biólogos y los conservacionistas no comunican de una forma más efectiva el por qué de la conservación.

Promover una cultura de leer y escribir en la enseñanza universitaria peruana. La escasez de revistas peer review editadas en los países tropicales y la falta de acceso a las revistas peer review editadas en los países desarrollados resultan en un circulo vicioso. Los estudiantes de biología en las universidades peruanas aún no reciben suficiente práctica o incentivo en leer, escribir y evaluar textos científicos. Una vez graduados, no tienen los recursos para enseñar a la próxima generación estas tareas tan básicas para cualquier científico. Las iniciativas para facilitar el acceso a las revistas de los países desarrollados sin duda ayudarán a romper este ciclo. Sin embargo, en nuestra opinión esta debilidad será superada sólo cuando las facultades de biología en las universidades peruanas establezcan la lectura, el escrito y la evaluación de los textos científicos como el corazón de sus programas.

Apoyar a los autores más prolíficos y a los centros de investigación más productivos en el trópico. Casi uno de cada cinco textos de Madre de Dios (y el 63\% de los textos relacionados a la ecología) se basan en los trabajos realizados desde el 1973 en la Estación Biológica Cocha Cashu, situada en el Parque Nacional del Manu. Más del 80\% de los 420 textos producidos por los investigadores de Cocha Cashu son artículos de revistas peer review, capítulos de libros, tesis y otras publicaciones de alta calidad. A pesar de ello, ni la estación, ni el parque tienen los fondos necesarios para una gestión eficiente, y tampoco han ganado el apoyo de fuentes nacionales de fondos científicos como el CONCYTEC y el IIAP.

Otras reformas provechosas. Para los individuos e instituciones de países desarrollados, otras reformas incluyen reducir el costo de acceso a sus revistas para los lectores de las zonas tropicales; publicar sus trabajos en revistas de acceso abierto; promover recursos para bibliotecas tropicales; y ofrecer incentivos profesionales a los científicos de países desarrollados quienes publican (o editan) artículos en revistas científicas tropicales.

Para los individuos e instituciones en el Perú, otras reformas incluyen establecer y apoyar por lo menos una revista peer review de conservación en cada región natural del país (como Ecología Aplicada de la UNALM o la Revista Peruana de Biología de la
UNMSM), enseñar a los estudiantes a usar software bibliográfico como EndNote o ProCite, obligar a los estudiantes a presentar versiones digitales de sus tesis, y eliminar textos "institucionales" cuyos autores son organizaciones y no individuos.

\section{Conclusiones}

Dado que los bosques amazónicos se están convirtiendo en tierras agrícolas a un paso vertiginoso, vale concluir con una reflexión acerca del record escrito que los biólogos y los conservacionistas de Madre de Dios hemos dejado del departamento hasta el momento. Inevitablemente, para un lector que hojea la bibliografía del departamento en el año 2104, lo que más llamará la atención dependerá del estado de conservación de Madre de Dios en ese futuro distante. Si las comunidades de plantas y animales siguen intactas y bien protegidas, entonces los temas que llamarán la atención son probablemente los mismos que hemos destacado arriba. Si, en cambio, la extinción de especies y la destrucción de bosques hayan transformado a Madre de Dios, entonces lo que probablemente llamará la atención del lector es la falta de investigación científica acerca de esa transformación, además de la evidente falta de comunicación entre los investigadores y los conservacionistas en nuestro tiempo. Al no dedicar mayores esfuerzos de compartir lo que hemos aprendido sobre la naturaleza tropical, nuestra generación corre el riesgo de perderla en una torre de Babel.

\section{Agradecimientos}

Agradecemos a todas las instituciones peruanas que abrieron las puertas de sus bibliotecas y base de datos, así como: L. Aranguena, M. Blácido, L. Benites, E. Gushiken, R. Leite Pitman, V. Miyakawa, E. Nanney, P. Rengifo Cardenas, M. Ruiz, M. Silman, A. Tovar y P. Vásquez. También agradecemos a las personas quienes revisaron los borradores de la bibliografía y de este manuscrito e hicieron adiciones, correcciones y comentarios: $\mathrm{M}$. Alexiades, D. Davidson, L. Emmons, J. Groenendijk, J. Janovec, C. Kirkby, R. Leite Pitman, P. Murphy, J. Ohl, O. Phillips, M. Tobler, R. von May y D. Yu. La compilación de la bibliografía fue financiada por una beca otorgada a N. C. A. P. del Amazon Conservation Association, la Asociación para la Conservación de la Cuenca Amazónica y la Fundación Gordon and Betty Moore. El diseño y la dirección de la página web de la bibliografía fueron dirigidos por J. Janovec y J. Best del Instituto de Investigación Botánica de Texas (BRIT) y financiados por una beca otorgada a J. Janovec de la Fundación Gordon and Betty Moore.

\section{Literatura citada}

Allen J. A. 1900. On mammals collected in southeastern Peru by Mr. H. H. Keays, with descriptions of new species. Bulletin of the American Museum of Natural History 13: 219-228.

Cieza de León P. 1877. Guerras civiles del Perú. Tomo primero: Guerra de las Salinas. Madrid: Administración García Rico y Compañía, Madrid.

Clark J. A. \& R. M. May. 2002a. How biased are we?: Even now, conservation research is still lopsided. Conservation in Practice 3: 28-29.

Clark J. A. \& R. M. May. 2002b. Taxonomic bias in conservation research. Science 10297: 191-192.

Dourojeanni M. J. 2003. Impactos socioambientales de las carreteras transfronterizas y fronterizas en los departamentos de Madre de Dios y Ucayali y capacidad de respuesta del Perú. In: R. Leite Pitman, N. Pitman, \& P. Álvarez, eds. Alto Purús: Biodiversidad, conservación y manejo. Center for Tropical Conservation, Lima. Pp. 237-246. 
Duellman W. E. 1978. The biology of an equatorial herpetofauna in Amazonian Ecuador. Miscellaneous Publications of the Museum of Natural History, University of Kansas 65: $1-352$.

Duellman W. E. 1988. Patterns of species diversity in anuran amphibians in the American tropics. Annals of the Missouri Botanical Garden 75: 79-104.

Fazey I., J. Fischer \& D. B. Lindenmayer. 2005a. What do conservation biologists publish? Biological Conservation 124: 63-73.

Fazey I., J. Fischer, \& D. B. Lindenmayer. 2005b. Who does all the research in conservation biology? Biodiversity and Conservation 14: 917-934.

Fazey I., J. G. Salisbury, D. B. Lindenmayer, J. Maindonald, \& R. Douglas. 2004. Can methods applied in medicine be used to summarize and disseminate 2conservation research? Environmental Conservation 31: 190-198.

Flaspohler D. J., B. R. Bub, \& B. A. Kaplin. 2000. Application of conservation biology 2research to management. Conservation Biology 14: 1898-1902.

Flores C. F., \& P. M. S. Ashton. 2000. Harvesting impact and economic value of Geonoma deversa, Arecaceae, an understory palm used for roof thatching in 5the Peruvian Amazon. Economic Botany 54: 267-277.

Gentry A. H. 1990. Four Neotropical rainforests. New Haven: Yale University Press.

Holmgren M. \& S. A. Schnitzer. 2004. Science on the rise in developing countries. PLoS Biology 2: 10-13.

Goulding M., C. Cañas, R. Barthem, B. Forsberg \& H. Ortega. 2003. Amazon headwaters: Rivers, wildlife, and conservation in southeastern Peru. Lima: Amazon Conservation Association.

INRENA(Instituto Nacional de Recursos Naturales) \& PRO-MANU (Proyecto Aprovechamiento y Manejo Sostenible de la Reserva de Biosfera y Parque Nacional del Manu). 2004. Plan maestro del Parque Nacional del Manu. INRENA y PRO-MANU, Cusco.

INRENA(Instituto Nacional de Recursos Naturales), FZS (Frankfurt Zoological Society), \& CDC-UNALM (Centro de Datos para la Conservación - Universidad Nacional Agraria La Molina). 2003. Hacia un sistema de monitoreo ambiental remoto estandarizado para el SINANPE: Informe final piloto 2002: Parque Nacional Bahuaja-Sonene, Reserva Nacional Tambopata y Reserva Comunal Amarakaeri. INRENA, FZS y CDC-UNALM, Lima.

INRENA(Instituto Nacional de Recursos Naturales), FZS (Frankfurt Zoological 2Society), \& CDC-UNALM (Centro de Datos para la Conservación - Universidad 2Nacional Agraria La Molina). 2004. Hacia un sistema de monitoreo ambiental remoto estandarizado para el SINANPE: Estudio de caso 2003: Parque Nacional Manu y Zona Reservada del Alto Purús. INRENA, FZS y CDC-2UNALM, Lima.

Janzen D. H., ed. 1983. Costa Rican natural history. Chicago: University of Chicago Press.

Kirkby C. 2002. Análisis inicial del impacto económico del turismo en Puerto Maldonado y periferia. Informe no publicado para el INRENA (Instituto Nacional 5de Recursos Naturales) y World Wildlife Fund-Perú.

Kirkby C. 2004. Manual metodológico para el monitoreo ambiental y socioeconómico de la Reserva de Biosfera del Manu. PRO-MANU (Proyecto Aprovechamiento y Manejo Sostenible de la Reserva de Biosfera y Parque Nacional del Manu), Cusco.

Kometter R. F., M. Martinez, A. G. Blundell, R. E. Gullison, M. K. Steininger, \& R. E. Rice. 2004. Impacts of unsustainable mahogany logging in Bolivia and Peru. Ecology and Society 9: 12 .
Leite Pitman M. R. P., N. C. A. Pitman, \& P. Álvarez, eds. 2003. Alto Purús: Biodiversidad, conservación y manejo. Lima: Center for Tropical Conservation.

ONERN (Oficina Nacional de Evaluacíon de Recursos Naturales). 1972. Inventario, evaluacíon e integracíon de los recursos naturales de la zona de los ríos Inambari y Madre de Dios. ONERN, Lima.

ONERN (Oficina Nacional de Evaluacíon de Recursos Naturales). 1977. Inventario, evaluacion e integracion de los recursos naturales de la zona Iberia-Iñapari. ONERN, Lima.

Ormerod S. J., N. D. Barlow, E. J. P. Marshall, \& G. Kerby. 2002. The uptake of applied ecology. Journal of Applied Ecology 39: 1-7.

Ortiz E. 2002. Brazil nut (Bertholletia excelsa). In: P. Shanley, A. Pierce, S. Laird, \& A. Guillen, eds. Tapping the green market: Certification and management of non-timber forests products. Earthscan Publications, London. Pp. 61-74.

Pautrat L. 2001. Análisis de amenazas y oportunidades para la conservación de la biodiversidad de la zona de conectividad ríos Manu-Tambopata. Informe no publicado para la Sociedad Peruana de Eco-desarrollo, Puerto Maldonado.

Pautrat L. 2003. Estudio preliminar: Extracción y comercio ilegal de productos forestales maderables, Madre de Dios, SE Perú, 2002-2003. Informe no publicado.

Peres C. A., C. Baider, P. A. Zuidema, L. H. O. Wadt, K. A. Kainer, et al. 2003. Demographic threats to the sustainability of Brazil nut 8exploitation. Science 302: 2112-2114.

Pitman N., D. K. Moskovits, W. S. Alverson, \& R. Borman A., eds. 2002. Ecuador: Serranías Cofán - Bermejo, Sinangoe. Chicago: The Field Museum.

Pitman N. C. A., M. del C. Loyola Azáldegui, K. Salas, G. Vigo T. \& D. A. Lutz. 2007. Written accounts of an Amazonian landscape over the last 450 years. Conservation Biology 21(1): 253-262.

Primack R. B. 2001. Publish again in another language. Conservation Biology 15: 290-15291.

PullinA. S., \& T. M. Knight. 2001. Effectiveness in conservation practice: Pointers from 17 medicine and public health. Conservation Biology 15: 50-54.

Pullin A. S., T. M. Knight, D. A. Stone, \& K. Charman. 2004. Do conservation managers use scientific evidence to support their decision-making? Biological Conservation 119: 245-252.

Schulte-Herbrüggen B. 2003. Tala ilegal en la Zona Reservada del Alto Purús, a lo largo del río de Las Piedras, Madre de Dios. In: R. Leite Pitman, N. Pitman, y P. Álvarez, eds. Alto Purús: Biodiversidad, conservación y manejo. Center for Tropical Conservation, Lima. Pp. 191-206.

Soulé M. E. 1985. What is conservation biology. Bioscience 35: 727-734.

Terborgh J. 1983. Five New World primates: A study in comparative ecology. Princeton: Princeton University Press.

Terborgh J. 1999. Requiem for nature. Washington, DC: Island Press.

Ulloa L., ed. 1899. Relación de la jornada y descubrimiento del Río Manu (hoy Madre de Dios) por Juan Álvarez Maldonado en 1567. Sevilla: Imp. y lit. de C. Salas.

Wahl L., \& K. Rummenhoeller 1991. La región de Madre de Dios: Bibliografía anotada. Cusco: Centro de Estudios Regionales Andinos Bartolomé de las Casas.

Yu D. W., T. Hendrickson, \& A. Castillo. 1997. Ecotourism and conservation in Amazonian Peru: Short-term and long-term challenges. Environmental Conservation 24: 130-138. 